\title{
Die Aktivierungsenergie für die Quergleitung aufgespaltener Sdhraubenversetzungen*
}

\author{
Von Helmut Wolf \\ Aus dem Institut für theoretische und angewandte Physik der Technischen Hochschule Stuttgart \\ und dem Max-Planck-Institut für Metallforschung, Stuttgart \\ (Z. Naturforschg. 15 a, 180-193 [1960] ; eingegangen am 31. Oktober 1959)
}

\begin{abstract}
Die Quergleitung von Schraubenversetzungen im kubisch-flächenzentrierten Gitter wird als einfacher Elementarprozeß mit eindeutigem Reaktionsweg und definiertem Sattelpunkt behandelt. Die Aktivierungsenergie für diesen Prozeß wird für die vorderste, unmittelbar an einer Lomer-CottrelLVersetzung anliegende Versetzung einer Versetzungsgruppe berechnet. Dazu wird das Spannungsfeld der Versetzungsgruppe in der primären Gleitebene und seine Wirkung auf die Aufspaltung der quergleitenden Versetzung untersucht. Die kritische Länge $2 l_{0}$, die den Sattelpunkt bestimmt, ergibt sich unter Berücksichtigung des Spannungsfeldes der Versetzungsgruppe in der Quergleitebene durch ein Variationsverfahren. Die Aktivierungsenergie setzt sich aus der Änderung der Selbst- und Wechselwirkungsenergie der beiden längs $2 l_{0}$ rekombinierenden Halbversetzungen, der Änderung der Stapelfehlerenergie und der von der äußeren Spannung geleisteten Arbeit zusammen. Die Versetzungsenergien werden für große Aufspaltungen mit Hilfe der linearen Kröserschen Theorie und für kleine Aufspaltungen an Hand des Peierlsschen Modells berechnet.

Das Ergebnis der Rechnung ist ein linearer Zusammenhang zwischen der Aktivierungsenergie $U$ und dem Logarithmus der äußeren Spannung $\tau$ für eine bestimmte Zahl $n$ von Versetzungen in der Versetzungsgruppe und für einen bestimmten Wert der spezifischen Stapelfehlerenergie $\gamma$. Der Proportionalitätsfaktor $A$ ist von $n$ wenig, von $\gamma$ stark abhängig. Der Vergleich mit dem Experiment ermöglicht die experimentelle Bestimmung der Stapelfehlerenergie von kubisch-flächenzentrierten Metallen.
\end{abstract}

Der Beginn des Bereichs III der Verfestigungskurve kubisch-flächenzentrierter Einkristalle wird auf die thermisch aktivierte Quergleitung von in Halbversetzungen aufgespaltenen Schraubenversetzungen zurückgeführt ${ }^{1,2}$. Der Logarithmus der Spannung $\tau_{\text {III }}$ nimmt linear mit wachsender Temperatur $\mathrm{ab}^{3-5}$ und steigt linear mit dem Logarithmus der Abgleitgeschwindigkeit an ${ }^{5}$. Um diese Zusammenhänge zu erklären, und um aus ihnen spezifische Stapelfehlerenergien experimentell $\mathrm{zu}$ bestimmen ${ }^{5}$, wird in der vorliegenden Arbeit die Aktivierungsenergie für die Quergleitung als Funktion der äußeren Spannung $\tau$ berechnet. Der Rechnung wird das von $\mathrm{S}_{\text {снӧоск }}{ }^{6}$ sowie $\mathrm{S}_{\text {снӧок }}$ und $\mathrm{S}_{\mathrm{EEgER}}{ }^{7}$ verwendete Modell für die Quergleitung zugrunde gelegt (Abb. 1). SсHÖсK und SeEger haben die Aktivierungsenergie für eine einzelne Versetzung als Funktion der Spannung in der Quergleitebene an Hand des Peierlsschen Modells berechnet, ohne dabei die Spannung in der primären Gleitebene zu berücksichtigen.

* Dissertation, TH Stuttgart 1959.

1 A. Seeger, J. Diehl, S. Mader u. H. Rebstock, Phil. Mag. 2, 323 [1957].

2 A. Seeger, Kristallplastizität, Handbuch der Physik VII/2, Springer-Verlag, Berlin-Göttingen-Heidelberg 1958.

3 P. Haasen, Phil. Mag. 3, 384 [1958].
In der vorliegenden Arbeit wird die Aktivierungsenergie $U$ für die Quergleitung der vordersten, an einer Lomer-Cottrell-Versetzung anliegenden Ver-

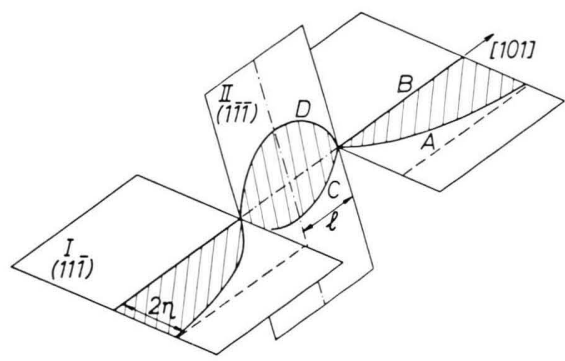

Abb. 1. Quergleitung einer aufgespaltenen Schraubenversetzung. $\mathrm{I}=$ primäre Gleitebene, $\mathrm{II}=$ Quergleitebene.

setzung einer Versetzungsgruppe in Abhängigkeit von der äußeren Spannung $\tau$, der spezifischen Stapelfehlerenergie $\gamma$ des bei der Aufspaltung sich zwischen den Halbversetzungen bildenden Stapelfehlerbandes und der Zahl $n$ der Versetzungen in der Gruppe bestimmt. Wir betrachten als Aktivierungs-

${ }^{4}$ R. Berner, Diplomarbeit, Stuttgart 1957.

5 A. Seeger, R. Berner u. H. Wolf, Z. Phys. 155, 247 [1959].

${ }^{6}$ G. Sснӧск, Dissertation, Stuttgart 1954.

7 G. Schöck u. A. Seeger, Rep. Conf. Defects Solids, Physical Society, London 1955, S. 340. 
energie die Energie, die unter Berücksichtigung der von der äußeren Spannung geleisteten Arbeit nötig ist, um die in Abb. 2 dargestellte Rekombination

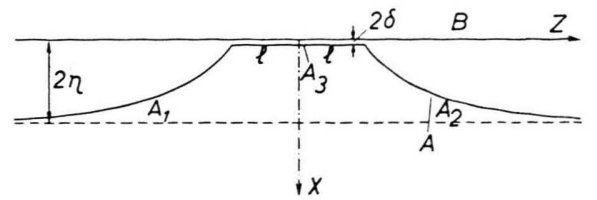

Abb. 2. Thermisch aktivierte Rekombination der beiden Halbversetzungen einer aufgespaltenen Schraubenversetzung in der primären Gleitebene.

der beiden Halbversetzungen der quergleitenden Versetzung über die Länge $2 l_{0}$ herbeizuführen. In $\S 1$ wird die in einer Versetzungsgruppe gegebene Ausgangsaufspaltung $2 \eta$ berechnet. Sie ist kleiner als die Aufspaltung $2 \eta_{0}$ einer einzelnen Versetzung, auf die keine Spannung wirkt. Die kritische Länge $2 l_{0}$ ist der Minimalbetrag der Länge $2 l$, der nötig ist, damit die Versetzung nach dem thermisch aktivierten Prozeß allein unter der Wirkung der äußeren Spannung in der Quergleitebene weiter wandern kann. Sie ist abhängig von der in der Quergleitebene wirkenden Spannung, die wir in $\S 2$ berechnen. Die kritische Länge $2 l_{0}$ wird in $\S 4$ bestimmt. In $\S 3$ werden die einzelnen Beiträge zur Aktivierungsenergie zusammengestellt. Die elastischen Selbstund Wechselwirkungsenergien der an der Reaktion beteiligten Halbversetzungsstücke entnehmen wir einer Arbeit von Kröner, SEEgER und Wolf ${ }^{8}$, in der sie mit dem von KRöNer ${ }^{9}$ entwickelten Verfahren im Rahmen der linearen Elastizitätstheorie berechnet werden. Für große Stapelfehlerenergien, d. h. kleine Aufspaltungen, ist die lineare Theorie unbrauchbar. Deshalb wurde für wenig aufgespaltene Versetzungen die Rechnung mit der auf Versetzungsgruppen erweiterten Methode von SEEger und $\mathrm{S}_{\mathrm{CHÖCK}}{ }^{6,7}$ durchgeführt, der das PeierLssche Modell zugrunde liegt ${ }^{10}$. Die Ergebnisse dieser Rechnung werden gemeinsam mit denen der linearen Theorie in $\S 5$ berichtet.

Das in der vorliegenden Arbeit verwendete Modell darf nur als eine Näherung betrachtet werden. In Wirklichkeit erfolgt die Quergleitung nicht, wie hier

8 E. Kröner, A. SEeger u. H. Wolf, Veröffentlichung demnächst.

๑ E. Kröner, Kontinuumstheorie der Versetzungen und Eigenspannungen, Springer-Verlag, Berlin-Göttingen-Heidelberg 1958, insbes. § 18.

$10 \mathrm{H}$. Wolf, unveröffentlicht. angenommen, auf einem eindeutigen Reaktionsweg mit definiertem Sattelpunkt, sondern es sind noch andere Wege für den thermisch aktivierten Prozeß mit kleinerer Aktivierungsenergie möglich ${ }^{11,12}$. SEEger, Berner und WolF ${ }^{5}$ haben gezeigt, daß diese Prozesse mit geringem statistischen Gewicht in die Gesamtreaktion eingehen, so daß das gewählte Modell eine brauchbare Näherung darstellt. Ein von F LeISCHER ${ }^{12}$ vorgeschlagenes Modell wird ebenfalls in $\S 5$ näher diskutiert.

\section{Die Aufspaltung der Versetzungen in einer Versetzungsgruppe}

Die Gleitebene der Versetzungsgruppe wird als $x z$-Ebene des Koordinatensystems gewählt. Die Versetzungsgruppe besteht aus $n$ geradlinigen, parallelen Schraubenversetzungen, die in $z$-Richtung verlaufen und unter der Schubspannung ${ }^{13}-\tau$ an einem Hindernis aufgestaut werden (Abb. 3). Eshelby,

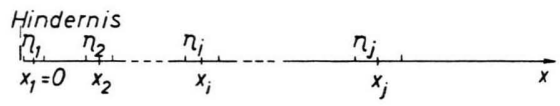

Abb. 3. An einem Hindernis aufgestaute Versetzungsgruppe aufgespaltener Schraubenversetzungen. Die Versetzungen verlaufen senkrecht zur Bildebene.

Frank und Nabarro ${ }^{14}$ haben für eine solche Anordnung nicht-aufgespaltener Versetzungen die Gleichgewichtslagen $x_{i}$ der einzelnen Versetzungen bestimmt. Sie fanden, daß diese durch die Nullstellen der ersten Ableitung des $n$-ten Laguerreschen Polynoms

$$
L_{n}^{\prime}(\bar{x})=\sum_{k=0}^{n-1} \frac{(-1)^{k+1}}{k !}\left(\begin{array}{c}
n \\
k+1
\end{array}\right) \bar{x}^{k}=0
$$

gegeben sind, wobei $\bar{x}=2 \tau x / B$ und $B=b G / 2 \pi$ gesetzt ist. $b$ ist der Abstand nächster Nachbarn im Gitter und $G$ der Schubmodul des Materials. In der Nähe des Koordinatenursprungs (Hindernis) sind diese Nullstellen näherungsweise durch

$$
\bar{x}_{i} \approx j_{i}{ }^{2} / 4 n
$$

gegeben, wobei $j_{i}$ die $i$-te Nullstelle der BEssel-Funktion $J_{1}$ ist. Für großes $n$ kann man die Verteilung

11 J. Friedel, Dislocations and Mechanical Properties of Crystals, J. Wiley \& Sons, New York 1957, S. 330.

12 R. L. Fleischer, Acta Met. 7, 134 [1959].

13 Wir bezeichnen mit $-\tau$ und $\tau_{x}$ die Komponenten $\sigma_{y z}$ und $\sigma_{x y}$ der äußeren Spannung.

14 J. D. Eshelby, F. C. Frank u. F. R. N. Nabarro, Phil. Mag. 42, 351 [1951]. 
der Wurzeln $\bar{x}_{i}$ durch die Dichtefunktion ${ }^{15}$

$$
D(\bar{x})=\frac{1}{2 \pi} \sqrt{(4 n-\bar{x}) / \bar{x}}
$$

beschreiben, wo $D(\bar{x})$ die Zahl der Versetzungen pro Längeneinheit auf der $\bar{x}$-Achse ist.

Die Gl. (1.1) gilt streng nur für nicht-aufgespaltene Versetzungen. Wir interessieren uns besonders dafür, wie die Aufspaltung $2 \eta_{1}$ der unmittelbar am Hindernis angepreßten Versetzung von $\tau, n$ und $\gamma$ abhängt. Die näherungsweise Berechnung erfolgt in drei Schritten:

1. Die Aufspaltung einer beliebigen, von der vordersten verschiedenen Versetzung wird berechnet unter der Annahme, daß alle anderen Versetzungen als nicht-aufgespalten betrachtet werden dürfen (\$1.1).

2. Die so errechneten Aufspaltungen geben aus zwei Gründen zu einer Verschiebung $\lambda_{i}$ der Gleichgewichtslagen der Versetzungen Anlaß:

a) Das Spannungsfeld jeder Versetzung weicht wegen der endlichen Aufspaltung von demjenigen einer nicht-aufgespaltenen Versetzung ab und erhält deshalb einen „Dipolanteil“. b) Die Kräfte auf die beiden Halbversetzungen einer vollständigen Versetzung sind nicht entgegengesetzt gleich, so daß eine Verschiebung der Gleichgewichtslage gegenüber der aus Gl. (1.1) berechneten auftritt ( $§ 1.2$ ).

3. Die Aufspaltung der vordersten, unmittelbar am Hindernis anliegenden Versetzung muß separat behandelt werden, da auf sie außer den $n-1$ übrigen Versetzungen und der äußeren Spannung auch das Hindernis wirkt. Eine erste Näherung von $\eta_{1}$ erhält man wie in $\S 1.1$. Die Berechnung der zweiten Näherung erfolgt unter Berücksichtigung der ersten Näherung der Aufspaltung $2 \eta_{i}$ und der Verschiebung $\lambda_{i}$ der Versetzungen. Im Prinzip kann man diese Schritte beliebig oft wiederholen. Wir begnügen uns mit der zweiten Näherung (§ 1.3).

\subsection{Die Aufspaltung $2 \eta_{j}$ in erster Näherung $(j \neq 1)$}

An der Stelle $x_{j}$ ist die Summe der Spannung der übrigen nicht-aufgespaltenen Versetzungen und der äußeren Spannung

$$
\sigma_{y z}\left(x_{j}\right)=-\tau+B \sum_{\substack{i=1 \\ i \neq j}}^{n} \frac{1}{x_{j}-x_{i}}=0 .
$$

Sie verschwindet nicht in der Umgebung von $x_{j}$ und übt deshalb auf die beiden Halbversetzungen eine Kraft ${ }^{16} K_{\mathrm{F}}$ aus, die die Aufspaltung zu verringern sucht. Ihr wirkt die Kraft $K_{\text {in }}$, die von der Wechselwirkung der beiden Halbversetzungen herrührt, entgegen. Die Aufspaltung erhält man, indem man

$$
K_{\mathrm{F}}+K_{\text {in }}=0
$$

setzt. Es ist zu beachten, daß die Burgers-Vektoren der Halbversetzungen außer der Schraubenkomponente $b / 2$ auch eine Stufenkomponente $b / 2 \sqrt{ } 3$ bzw. $-b / 2 \sqrt{3}$ besitzen, auf die die Spannungskomponente $\tau_{x}$ die Kraft

$$
K_{x}=\frac{b}{2 \sqrt{3}} \tau_{x}
$$

ausübt.

Die Spannung $\sigma_{y z}$ an der Stelle $x=x_{j}+\Delta x_{j}$ ist durch

$$
\begin{aligned}
& \sigma_{y z}(x)=-\tau+B \sum_{\substack{i=1 \\
i \neq j}}^{n} \frac{1}{x-x_{i}} \\
& =-\tau+B \sum_{\substack{i=1 \\
i \neq j}}^{n}\left(\frac{1}{x_{j}-x_{i}}-\frac{\Delta x_{j}}{\left(x_{j}-x_{i}\right)^{2}}+\frac{\left(\Delta x_{j}\right)^{2}}{\left(x_{j}-x_{i}\right)^{3}}-+\ldots\right)
\end{aligned}
$$

gegeben. Unter Berücksichtigung von Gl. (1.4) erhält man

$$
\sigma_{y z}\left(x_{j}+\Delta x_{j}\right)=-B Q_{j} \Delta x_{j}+B R_{j}\left(\Delta x_{j}\right)^{2}, j \neq 1,
$$

wobei

$$
Q_{j}=\sum_{\substack{i=1 \\ i \neq j}}^{n} \frac{1}{\left(x_{j}-x_{i}\right)^{2}} \quad \text { und } \quad R_{j}=\sum_{\substack{i=1 \\ i \neq j}}^{n} \frac{1}{\left(x_{j}-x_{i}\right)^{3}}
$$

bedeutet. Die Kräfte

$$
K_{\mathrm{F}}^{\prime}=\frac{1}{2} b \sigma_{y z}\left(x_{j} \mp \eta_{j}\right)= \pm \frac{1}{2} b B Q_{j} \eta_{j}+\frac{1}{2} b B R_{j} \eta_{j}^{2}
$$

auf die beiden Halbversetzungen sind nicht entgegengesetzt gleich. Das hat zur Folge, daß sich die aufgespaltene Versetzung aus der Lage $x_{j}$ verschiebt. Im Gleichgewicht ist dann $K_{\mathrm{F}}$ näherungsweise durch

$$
K_{\mathrm{F}}=\frac{1}{2} b B Q_{j} \eta_{j}+K_{x}
$$

gegeben.

Aus der Wechselwirkungsenergie ${ }^{9}$

$E_{\mathrm{w}}=\frac{G b^{2}}{8 \pi}\left(\ln \frac{L}{\eta_{j}}-1-\frac{\mu}{3}\left(\ln \frac{L}{\eta_{j}}-\frac{1}{2}\right)\right), \quad \mu=\frac{1}{1-v}$

15 G. Leibfried, Z. Phys. 130, 214 [1951].

${ }^{16} K$ bezeichnet Kräfte pro Längeneinheit der Versetzungen, die die Aufspaltung bestimmen; $\tilde{K}$ bezeichnet Kräfte pro Längeneinheit der Versetzungen, die die Änderung der Gleichgewichtslagen bestimmen. 
( $v$ ist die Porssonsche Konstante und $2 L$ die Länge der Versetzung) und der Stapelfehlerenergie

$$
E_{\mathrm{St}}=2 \eta_{j} \gamma
$$

erhält man durch Differentiation nach $2 \eta_{j}$ die innere Kraft

$$
K_{\text {in }}=\frac{\mathrm{d}\left(E_{\mathrm{w}}+E_{\mathrm{St}}\right)}{\mathrm{d}\left(2 \eta_{j}\right)}=\gamma-\frac{b B}{8}\left(1-\frac{\mu}{3}\right) \frac{1}{\eta_{j}} .
$$

(1.11) und (1.14) in (1.5) eingesetzt ergibt

$$
\gamma+K_{x}+\frac{b}{2} B Q_{j} \eta_{j}-\frac{b B}{8}\left(1-\frac{\mu}{3}\right) \frac{1}{\eta_{j}}=0
$$

und daraus folgt

$\eta_{j}=\frac{\gamma+K_{x}}{b B Q_{j}}+\sqrt{\left(\frac{\gamma+K_{x}}{b B Q_{j}}\right)^{2}+\frac{1}{4}\left(1-\frac{\mu}{3}\right) \frac{1}{Q_{j}}}, \quad j \neq 1$.

\subsection{Die Verschiebung $\lambda_{i}$ der aufgespaltenen Versetzungen}

Gl. (1.10) zeigt, daß, da das Spannungsfeld um $x_{j}$ nicht symmetrisch zu $x_{j}$ ist, auf die Gesamtversetzung $j$ eine resultierende Kraft wirkt, die eine Verschiebung gegen die durch Gl. (1.1) gegebene Gleichgewichtslage $x_{j}$ zur Folge hat. Außerdem bewirkt die Aufspaltung der übrigen Versetzungen eine Änderung des Spannungsfeldes. Wir berechnen zunächst diese „Dipolkorrektur“ für Schrauben- und Stufenanteil der Halbversetzungen und bestimmen die neue Gleichgewichtslage, indem wir die Gesamtkraft des korrigierten Feldes Null setzen.

Es wird

$$
\lambda_{1}=0
$$

gesetzt und damit der Ursprung des Koordinatensystems in die Mitte der Versetzung 1 gelegt.

Schraubenanteil. An der Stelle $x$ ist die Schubspannung $\sigma_{y z i}$ der $i$-ten Versetzung

$$
\sigma_{y z i}=\frac{B}{2}\left(\frac{1}{x-x_{i}+\eta_{i}}+\frac{1}{x-x_{i}-\eta_{i}}\right) \text {. }
$$

Entwickelt man Gl. (1.18) nach $\eta_{i}$, so erhält man

$$
\sigma_{y z i}=B\left(\frac{1}{x-x_{i}}+\frac{\eta_{i}{ }^{2}}{\left(x-x_{i}\right)^{3}}\right)
$$

und an der Stelle $x_{j} \pm \eta_{j}$ die Dipolkorrektur

$$
\sigma_{y z i D}=B \frac{\eta i^{2}}{\left(x-x_{i}\right)^{3}} \approx B \frac{\eta_{i}{ }^{2}}{\left(x_{j}-x_{i}\right)^{3}} .
$$

Summation über $i$ ergibt die Gesamtkorrektur des Schraubenanteils

$$
\sigma_{y z D}=B \sum_{\substack{i=1 \\ i \neq j}}^{n} \frac{\eta_{i}{ }^{2}}{\left(x_{j}-x_{i}\right)^{3}},
$$

die auf die $j$-te Versetzung die Kraft

$$
\tilde{K}_{z j D}=b B \sum_{\substack{i=1 \\ i \neq j}}^{n} \frac{\eta i^{2}}{\left(x_{j}-x_{i}\right)^{3}}
$$

ausübt.

Stufenanteil. Die Schubspannung $\sigma_{x y i}$ der $i$-ten Versetzung ist an der Stelle $x$

$$
\begin{gathered}
\sigma_{x y i}=B_{x}\left(\frac{1}{x-x_{i}+\eta_{i}}-\frac{1}{x-x_{i}-\eta_{i}}\right), \quad B_{x}=\frac{b_{x} G}{2 \pi(1-v)}, \\
b_{x}=\frac{b}{2 \sqrt{3}} .
\end{gathered}
$$

(1.23) nach $\eta_{i}$ entwickelt ergibt

$$
\sigma_{x y i}=-2 B_{x} \frac{\eta_{i}}{\left(x-x_{i}\right)^{2}} .
$$

Diese Spannung übt auf die $j$-te Versetzung die Kraft $\tilde{K}_{x j i}=-2 b_{x} B_{x} \eta_{i}\left(\frac{1}{\left(x_{j}-\eta_{j}-x_{i}\right)^{2}}-\frac{1}{\left(x_{j}+\eta_{j}-x_{i}\right)^{2}}\right)$

aus, aus der durch Entwicklung nach $\eta_{j}$ und Summation über $i$ die Gesamtkraft

$$
\tilde{K}_{x j D}=-8 b_{x} B_{x} \eta_{j} \sum_{\substack{i=1 \\ i \neq j}}^{n} \frac{\eta_{i}}{\left(x_{j}-x_{i}\right)^{3}}
$$

des Stufenanteils folgt. Schrauben- und Stufenanteil ergeben die Gesamtkorrektur

$$
\begin{aligned}
\tilde{K}_{j D} & =\tilde{K}_{z j D}+\tilde{K}_{x j D} \\
& =b B \sum_{\substack{i=1 \\
i \neq j}}^{n} \frac{\eta_{i}{ }^{2}}{\left(x_{j}-x_{i}\right)^{3}}-8 b_{x} B_{x} \eta_{j} \sum_{\substack{i=1 \\
i \neq j}}^{n} \frac{\eta_{i}}{\left(x_{j}-x_{i}\right)^{3}} .
\end{aligned}
$$

Ist die Gleichgewichtslage nach der Verschiebung $x_{j}-\Delta x_{j 1}$ für die linke und $x_{j}+\Delta x_{j 2}$ für die rechte Halbversetzung $\left(\Delta x_{j 1}+\Delta x_{j 2}=2 \eta_{j}\right)$, so ist mit den Gln. (1.8) und (1.27) die Gesamtkraft auf die j-te Versetzung, die im Gleichgewicht verschwindet, durch

$$
\begin{aligned}
\tilde{K}_{j}=b B\left(R_{j}\left(\Delta x_{j 2}\right)^{2}-\right. & \left(Q_{j}+2 \eta_{j} R_{j}\right) \Delta x_{j 2} \quad \\
& \left.+\eta_{j}\left(Q_{j}+2 \eta_{j} R_{j}\right)\right)+\tilde{K}_{j D}=0
\end{aligned}
$$

gegeben. Aus (1.28) ergibt sich

$$
\Delta x_{j 2}=\frac{1}{2}\left(\frac{Q_{j}}{R_{j}}+2 \eta_{j}-\sqrt{\left(\frac{Q_{j}}{R_{j}}\right)^{2}-\left(\begin{array}{r}
\left.4 \eta_{j}^{2}+\frac{4 \tilde{K}_{j D}}{b B R_{j}}\right) \\
j \neq 1 \quad(1.29)
\end{array}\right)}\right.
$$

und mit $\lambda_{j}=\Delta x_{j 2}-\eta_{j}$ die Verschiebung

$$
\begin{array}{r}
\lambda_{j}=\frac{1}{2}\left(\frac{Q_{j}}{R_{j}}-\sqrt{\left(\frac{Q_{j}}{R_{j}}\right)^{2}-4\left(\eta_{j}{ }^{2}+\frac{\tilde{K}_{j D}}{b B R_{j}}\right)}\right) \\
\approx \frac{R_{j}}{Q_{j}}\left(\eta_{j}{ }^{2}+\frac{\tilde{K}_{j D}}{b B R_{j}}\right), \quad j \neq 1,
\end{array}
$$

$\lambda_{1}=0 \quad$ [siehe Gl. (1.17)]. 


\subsection{Die Aufspaltung der Versetzung 1}

Da auf die vorderste Versetzung außer den $n-1$ übrigen Versetzungen und der äußeren Spannung auch das Hindernis wirkt, muß sie gesondert behandelt werden. Die linke Halbversetzung der Versetzung 1 wird am Hindernis aufgehalten. Es wird angenommen, daß das Spannungsfeld des Hindernisses eine so kurze Reichweite hat, daß es nur auf diese Halbversetzung wirkt.

a) Die erste Näherung von $\eta_{1}$

Die Berechnung der ersten Näherung von $\eta_{1}$ erfolgt wie bei den anderen Versetzungen $(\S 1.1)$, indem aus dem Spannungsfeld der übrigen nicht-aufgespaltenen Versetzungen an der Stelle $x=\eta_{1}$

$\sigma_{y z}=-\tau+B \sum_{i=\underline{2}}^{n} \frac{1}{\eta_{1}-x_{i}}$

$$
=-\tau-B\left(P_{1}+\eta_{1} Q_{1}-\eta_{1}^{2} R_{1}\right), \quad P_{1}=\sum_{i=2}^{n} \frac{1}{x_{i}}
$$

die $\mathrm{Kraft}^{17}$

$$
K_{\mathrm{F}}=\frac{b}{2}\left(\tau+B P_{1}+B Q_{1} \eta_{1}\right)+K_{x}
$$

bestimmt und die Summe

$$
\begin{aligned}
K_{\text {in }}+K_{\mathrm{F}}=\gamma & -\frac{b B}{8}\left(1-\frac{\mu}{3}\right) \frac{1}{\eta_{1}} \\
& +\frac{b}{2}\left(\tau+B P_{1}\right)+K_{x}+\frac{b}{2} B Q_{1} \eta_{1}=0
\end{aligned}
$$

gesetzt wird. Das ergibt

$$
\begin{aligned}
\eta_{1}=- & \frac{\frac{1}{2} b\left(\tau+B P_{1}\right)+K_{x}+\gamma}{b B Q_{1}} \\
& +\sqrt{\left(\frac{\frac{1}{2} b\left(\tau+B P_{1}\right)+K_{x}+\gamma}{b B Q_{1}}\right)^{2}+\frac{1}{4}\left(1-\frac{\mu}{3}\right) \frac{1}{Q_{1}}} .
\end{aligned}
$$

b) Die zweite Näherung von $\eta_{1}$

Unter Berücksichtigung der Aufspaltung $2 \eta_{i}$ und der Verschiebung $\lambda_{i}$ der Versetzungen wird die Rechnung von a) wiederholt.

Schraubenanteil. Die Schubspannung $\sigma_{y z i}$ der $i$-ten Versetzung ist an der Stelle $x$

$$
\sigma_{y z i}=\frac{B}{2}\left(\frac{1}{x-x_{i}-\lambda_{i}+\eta_{i}}+\frac{1}{x-x_{i}-\lambda_{i}-\eta_{i}}\right) .
$$

Durch Reihenentwicklung ergibt sich für $x=\eta_{1}$

$\sigma_{y z i}=-B\left(\frac{1}{x_{i}}+\frac{\eta_{1}-\lambda_{i}}{x_{i}{ }^{2}}+\frac{\left(\eta_{1}-\lambda_{i}\right)^{2}+\eta_{i}{ }^{2}}{x_{i}{ }^{3}}\right)$.

17 Die numerische Rechnung zeigt, daß zur Bestimmung der ersten Näherung das Glied mit $\eta_{1}{ }^{2}$ vernachlässigt werden darf.
Die Gesamtkomponente des Schraubenanteils erhält man durch Summation über $i$,

$\sigma_{y z}=-\tau-B\left(P_{1}+\eta_{1} Q_{1}+\sum_{i=2}^{n}\left(-\frac{\lambda_{i}}{x_{i}{ }^{2}}+\frac{\left(\eta_{1}-\lambda_{i}\right)^{2}+\eta_{i}{ }^{2}}{x_{i}{ }^{3}}\right)\right)$

Stufenanteil. Die Schubspannung

$$
\sigma_{x y i}=B_{r .}\left(\frac{1}{x-x_{i}-\lambda_{i}+\eta_{i}}-\frac{1}{x-x_{i}-\lambda_{i}-\eta_{i}}\right)
$$

wird für $x=\eta_{1}$ in eine Reihe entwickelt. Das ergibt

$$
\sigma_{x y i}=-2 B_{x} \eta_{i}\left(\frac{1}{x_{i}^{2}}+\frac{2\left(\eta_{1}-\lambda_{i}\right)}{x_{i}^{3}}\right) .
$$

Die Gesamtkomponente des Stufenanteils erhält man durch Summation über $i$,

$$
\sigma_{x y}=\tau_{x}-2 B_{x} \sum_{i=2}^{n}\left(\frac{\eta_{i}}{x_{i}{ }^{2}}+\frac{2\left(\eta_{1}-\lambda_{i}\right) \eta_{i}}{x_{i}{ }^{3}}\right) .
$$

Damit wird die Kraft

$$
K_{\mathrm{F}}=\frac{b}{2} B Q_{1} \eta_{1}+\frac{b}{2}\left(\tau+B P_{1}\right)+K_{x}+
$$

$$
\begin{aligned}
+\frac{b}{2} B \sum_{i=2}^{n}( & -\frac{\lambda_{i}}{x_{i}{ }^{2}}+\frac{\left(\eta_{1}-\lambda_{i}\right)^{2}+\eta_{i}{ }^{2}}{x_{i}{ }^{3}} \\
& \left.-\frac{2 B_{x}}{\sqrt{3} B}\left(\frac{\eta_{i}}{x_{i}{ }^{2}}+\frac{2\left(\eta_{1}-\lambda_{i}\right) \eta_{i}}{x_{i}{ }^{3}}\right)\right)
\end{aligned}
$$

oder

$$
K_{\mathrm{F}}=\frac{b}{2} B Q_{1} \eta_{1}+M \text {. }
$$

Im Gleichgewicht ist

$$
K_{\text {in }}+K_{\mathrm{F}}=\gamma-\frac{b B}{8}\left(1-\frac{\mu}{3}\right) \frac{1}{\eta_{1}}+\frac{b}{2} B Q_{1} \eta_{1}+M=0
$$

und

$\eta_{1}=-\frac{M+\gamma}{b B Q_{1}}+\sqrt{\left(\frac{M+\gamma}{b B Q_{1}}\right)^{2}+\frac{1}{4}\left(1-\frac{\mu}{3}\right) \frac{1}{Q_{1}}}$

In

$$
\begin{aligned}
& M=\frac{b}{2}\left(\tau+B P_{1}\right)+K_{x}+\frac{b}{2} B \sum_{i=2}^{n} \\
& \cdot\left(-\frac{\lambda_{i}}{x_{i}{ }^{2}}+\frac{\left(\eta_{1}-\lambda_{i}\right)^{2}+\eta_{i}{ }^{2}}{x_{i}{ }^{3}}-\frac{2 B_{x}}{\sqrt{ } B}\left(\frac{\eta_{i}}{x_{i}{ }^{2}}+\frac{2 \eta_{i}\left(\eta_{1}-\lambda_{i}\right)}{x_{i}{ }^{3}}\right)\right)
\end{aligned}
$$

ist für $\eta_{1}$ die erste Näherung einzusetzen.

\subsection{Die Berechnung von $P_{1}, Q_{j}$ und $R_{j}$}

Wir setzen

$$
P_{1}=\sum_{i=2}^{n} \frac{1}{x_{i}}=\alpha \sum_{i=2}^{n} \frac{1}{\bar{x}_{i}}=\alpha P_{1}, \quad \bar{x}_{i}=\alpha x_{i}, \quad \alpha=\frac{2 \tau}{B}
$$


und teilen die Summe $\bar{P}_{1}$ in zwei Teile auf,

$$
\bar{P}_{1}=\sum_{i=2}^{4} \frac{1}{\bar{x}_{i}}+\sum_{i=5}^{n} \frac{1}{\bar{x}_{i}}=\bar{P}_{1 \mathrm{I}}+\bar{P}_{1 \mathrm{II}} \text {. }
$$

$P_{1 \mathrm{II}}$ wird direkt mit Hilfe von Gl. (1.2) berechnet, $\bar{P}_{1 \mathrm{II}}$ als Integral mit Hilfe von Gl. (1.3),

$P_{1 \mathrm{II}}=\sum_{i=5}^{n} \frac{1}{\bar{x}_{i}}=\int_{\frac{1}{2}\left(\overline{x_{4}}+x_{5}\right)}^{4 n} \frac{D(\bar{x}) \mathrm{d} \bar{x}}{\bar{x}}=\frac{1}{2 \pi} \int_{\substack{\frac{1}{2}\left(\overline{x_{4}}+x_{\mathrm{s}}\right) \\ \bar{x}}}^{4 n} \sqrt{\frac{4 n-\bar{x}}{\bar{x}}} \mathrm{~d} \bar{x}$.

Das ergibt

$$
\begin{gathered}
P_{1 \mathrm{II}}=\frac{1}{4}\left(\sqrt{\frac{32 n^{2}}{j_{4}{ }^{2}+j_{5}{ }^{2}}}-1-\operatorname{arctg} \sqrt{\frac{32 n^{2}}{j_{4}{ }^{2}+j_{5}{ }^{2}}-1}\right), \\
P_{1 \mathrm{I}}=4 n \sum_{i=2}^{4} \frac{1}{j_{i}{ }^{2}} .
\end{gathered}
$$

Die Berechnung von $Q_{j}$ erfolgt analog zu der von $P_{1}$. Man setzt

$$
Q_{j}=\sum_{\substack{i=1 \\ i \neq j}}^{n} \frac{1}{\left(x_{j}-x_{i}\right)^{2}}=\alpha^{2} \sum_{\substack{i=1 \\ i \neq j}}^{n} \frac{1}{\left(\bar{x}_{j}-\bar{x}_{i}\right)^{2}}=\alpha^{2} Q_{j}
$$

und teilt die Summe in zwei Teile auf,

$$
\bar{Q}_{j}=\sum_{\substack{i=1 \\ i \neq j}}^{4} \frac{1}{\left(\bar{x}_{j}-\bar{x}_{i}\right)^{2}}+\sum_{i=5}^{n} \frac{1}{\left(\bar{x}_{j}-\bar{x}_{i}\right)^{2}}=Q_{j \mathrm{I}}+\bar{Q}_{j \mathrm{II}} .
$$

$Q_{j \mathrm{I}}$ wird direkt berechnet, $Q_{j \mathrm{II}}$ mit Hilfe der LEIBFRIEDschen Dichtefunktion,

$$
Q_{j \mathrm{II}}=\int_{\frac{1}{2}\left(x_{4}+\bar{x}_{5}\right)}^{4 n} \frac{D(\bar{x}) \mathrm{d} \bar{x}}{\left(\bar{x}_{j}-\bar{x}\right)^{2}}=\frac{1}{2 \pi} \int_{\substack{\left.\frac{1}{2}\left(x_{4}+x_{5}\right) \\ \bar{x}_{j}-\bar{x}\right)^{2}}}^{4 n} \sqrt{\frac{4 n-\bar{x}}{\bar{x}}} \mathrm{~d} \bar{x} .
$$

Das ergibt $Q_{j \mathrm{II}}=\frac{16 n^{2}}{\pi j_{j}^{4}}\left(\frac{\sqrt{\frac{32 n^{2}}{j_{4}{ }^{2}+j_{5}{ }^{2}}-1}}{8 n\left(\frac{1}{j_{j}{ }^{2}}-\frac{2}{j_{4}{ }^{2}+j_{5}{ }^{2}}\right)}-\frac{n}{\sqrt{\frac{16 n}{j_{j}{ }^{2}}-1}} \ln \frac{\sqrt{\frac{16 n^{2}}{j_{j}^{2}}-1}+\sqrt{\frac{32 n^{2}}{j_{4}{ }^{2}+j_{5}{ }^{2}}-1}}{\sqrt{\frac{16 n^{2}}{j_{j}{ }^{2}}-1}-\sqrt{\frac{32 n^{2}}{j_{4}{ }^{2}+j_{5}{ }^{2}}-1}}\right), \quad j \neq 1$,

$$
Q_{j \mathrm{I}}=16 n^{2} \sum_{\substack{i=1 \\ i \neq j}}^{4} \frac{1}{\left(j^{2}-j_{i}^{2}\right)^{2}} .
$$

$Q_{1}$ wird separat berechnet,

$$
Q_{1 \mathrm{II}}=\frac{1}{2 \pi} \int_{\frac{1}{2}\left(x_{4}+x_{5}\right)}^{4 n} \frac{1}{\bar{x}^{2}} \sqrt{\frac{4 n-\bar{x}}{\bar{x}}} \mathrm{~d} \bar{x}=-\frac{1}{8 \pi n} \int_{\substack{1 \\ \frac{1}{2}\left(x_{4}+\bar{x}_{5}\right)}}^{4 n} \sqrt{\frac{4 n}{\bar{x}}}-1\left(-\frac{4 n}{\bar{x}^{2}} \mathrm{~d} \bar{x}\right) .
$$

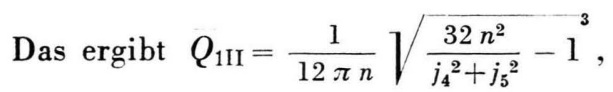

$$
\bar{Q}_{1 \mathrm{I}}=16 n^{2} \sum_{i=2}^{4} \frac{1}{j_{i}{ }^{4}} \text {. }
$$

Zur Berechnung von $R_{j}$ genügt es, die ersten Glieder der Reihe zu berücksichtigen. Man setzt

$$
R_{j}=\sum_{\substack{i=1 \\ i \neq j}}^{n} \frac{1}{\left(x_{j}-x_{i}\right)^{3}}=\alpha^{3} \sum_{\substack{i=1 \\ i \neq j}}^{n} \frac{1}{\left(\bar{x}_{j}-\bar{x}_{i}\right)^{3}}=\alpha^{3} R_{j}
$$

und erhält

$$
R_{j}=64 n^{3} \sum_{\substack{i=1 \\ i \neq j}}^{5} \frac{1}{\left(j_{j}^{2}-j i^{2}\right)^{3}} .
$$

In Abb. 4 ist die in diesem Abschnitt berechnete Aufspaltung für $j=1,2,3$ in Abhängigkeit von der äußeren Spannung für $n=20$ dargestellt. Für $v$ wurde der bei Kupfer unter näherungsweiser Berücksichtigung der Anisotropie ${ }^{18}$ gewonnene Wert verwendet.

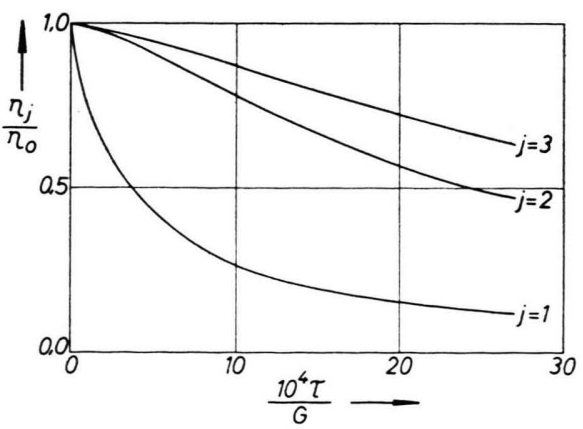

Abb. 4. Die Aufspaltung der Versetzungen 1, 2 und 3 in einer Versetzungsgruppe als Funktion der äußeren Spannung.

\section{Die Schubspannung in der Quergleitebene}

Die Schubspannung, die von den Versetzungen 2 bis $n$ der Versetzungsgruppe in der Quergleitebene hervorgerufen wird, bestimmt man, indem man die

18 A. SeEger u. G. Schöck, Acta Met. 1, 519 [1953]. 
Spannungskomponenten dieser Versetzungen im $x y z$-System $(x z$-Ebene $=$ primäre Gleitebene $)$ berechnet und in das $x^{\prime} y^{\prime} z^{\prime}$-System mit der $x^{\prime} z^{\prime}$-Ebene als Quergleitebene $\left(z=z^{\prime}\right.$, Abb. 5) transformiert.

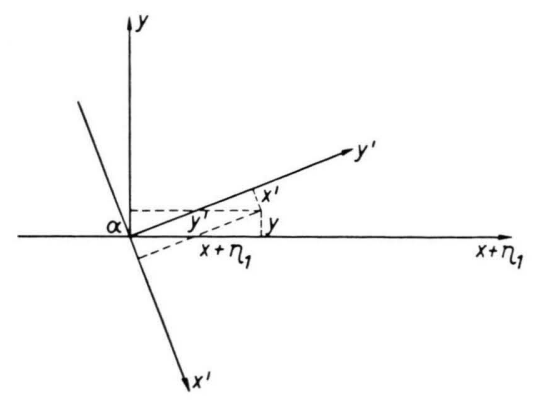

Abb. 5. Koordinatentransformation. $\quad x z$-Ebene $=$ primäre Gleitebene, $x^{\prime} z^{\prime}$-Ebene $=$ Quergleitebene. Die $z$-Achse steht senkrecht auf der Bildebene $\left(z=z^{\prime}\right)$.

Die Spannungskomponenten $\sigma_{z x}$ und $\sigma_{y z}$ lauten für eine vollständige Schraubenversetzung an der Stelle ${ }^{19} x_{i}$

$\sigma_{z x i}=-B \frac{y}{\left(x-x_{i}\right)^{2}+y^{2}}$ und $\sigma_{y z i}=B \frac{x-x_{i}}{\left(x-x_{i}\right)^{2}+y^{2}}$

Die Versetzungen sind um $\lambda_{i}$ verschoben und aufgespalten, folglich ist

$$
\sigma_{z x i}=-\frac{B}{2} y\left(\frac{1}{\left(x-x_{i}-\lambda_{i}+\eta_{i}\right)^{2}+y^{2}}\right.
$$

und

$$
\left.+\frac{1}{\left(x-x_{i}-\lambda_{i}-\eta_{i}\right)^{2}+y^{2}}\right)
$$

$\sigma_{y z i}=\frac{B}{2}\left(\frac{x-x_{i}-\lambda_{i}+\eta_{i}}{\left(x-x_{i}-\lambda_{i}+\eta_{i}\right)^{2}+y^{2}}+\frac{x-x_{i}-\lambda_{i}-\eta_{i}}{\left(x-x_{i}-\lambda_{i}-\eta_{i}\right)^{2}+y^{2}}\right)$.

Die Spannungskomponenten ${ }^{20}$ einer vollständigen Stufenversetzung an der Stelle $x=0$ sind

$$
\begin{array}{ll}
\sigma_{x x}=-B_{1} y \frac{3 x^{2}+y^{2}}{\left(x^{2}+y^{2}\right)^{2}}, & \sigma_{x y}=B_{1} x \frac{x^{2}-y^{2}}{\left(x^{2}+y^{2}\right)^{2}}, \\
\sigma_{y y}=B_{1} y \frac{x^{2}-y^{2}}{\left(x^{2}+y^{2}\right)^{2}}, & B_{1}=\frac{b G}{2 \pi} \frac{1}{1-v} .
\end{array}
$$

Damit wird die Schubspannungskomponente $\sigma_{x x i}$ der aufgespaltenen Versetzung an der Stelle $x_{i}$

$$
\begin{array}{r}
\sigma_{x x i}=-B_{x} y\left(\frac{3\left(x-x_{i}-\lambda_{i}+\eta_{i}\right)^{2}+y^{2}}{\left[\left(x-x_{i}-\lambda_{i}+\eta_{i}\right)^{2}+y^{2}\right]^{2}}\right. \\
\left.-\frac{3\left(x-x_{i}-\lambda_{i}-\eta_{i}\right)^{2}+y^{2}}{\left[\left(x-x_{i}-\lambda_{i}-\eta_{i}\right)^{2}+y^{2}\right]^{2}}\right) .
\end{array}
$$

\footnotetext{
19 A. SEeger, Theorie der Gitterfehlstellen, Handbuch der Physik VII/1, Springer-Verlag, Berlin-Göttingen-Heidelberg 1955.

${ }^{20} \sigma_{z z}$ wird nicht gebraucht.
}

Gl. (2.5) wird nach $\eta_{i}$ entwickelt; man erhält

$$
\sigma_{x x i}=4 B_{x} y \frac{\left(x-x_{i}-\lambda_{i}\right)\left[3\left(x-x_{i}-\lambda_{i}\right)^{2}-y^{2}\right]}{\left[\left(x-x_{i}-\lambda_{i}\right)^{2}+y^{2}\right]^{3}} \eta_{i} .
$$

In gleicher Weise ergeben sich

$\sigma_{y y i}=-4 B_{x} y \frac{\left(x-x_{i}-\lambda_{i}\right)\left[\left(x-x_{i}-\lambda_{i}\right)^{2}-3 y^{2}\right]}{\left[\left(x-x_{i}-\lambda_{i}\right)^{2}+y^{2}\right]^{3}} \eta_{i}$

und

$\sigma_{x y i}=-2 B_{x} \frac{\left(x-x_{i}-\lambda_{i}\right)^{4}+y^{4}-6\left(x-x_{i}-\lambda_{i}\right)^{2} y^{2}}{\left[\left(x-x_{i}-\lambda_{i}\right)^{2}+y^{2}\right]^{3}} \eta_{i}$.

Durch Summation über $i$ erhält man die Gesamtkomponenten im ungestrichenen System,

$$
\begin{array}{r}
\sigma_{z x}=-\frac{B}{2} y \sum_{i=2}^{n}\left(\frac{1}{\left(x-x_{i}-\lambda_{i}+\eta_{i}\right)^{2}+y^{2}}\right. \\
\left.+\frac{1}{\left(x-x_{i}-\lambda_{i}-\eta_{i}\right)^{2}+y^{2}}\right),
\end{array}
$$$$
\left.\sigma_{y z}=\frac{B}{2} \sum_{i=2}^{n} \frac{x-x_{i}-\lambda_{i}+\eta_{i}}{\left(x-x_{i}-\lambda_{i}+\eta_{i}\right)^{2}+y^{2}}+\frac{x-x_{i}-\lambda_{i}-\eta_{i}}{\left(x-x_{i}-\lambda_{i}-\eta_{i}\right)^{2}+y^{2}}\right) \text {, }
$$$$
\sigma_{x x}=4 B_{x} y \sum_{i=2}^{n} \frac{\left(x-x_{i}-\lambda_{i}\right)\left[3\left(x-x_{i}-\lambda_{i}\right)^{2}-y^{2}\right]}{\left[\left(x-x_{i}-\lambda_{i}\right)^{2}+y^{2}\right]^{3}} \eta_{i},
$$

$\sigma_{y y}=-4 B_{x} y \sum_{i=2}^{n} \frac{\left(x-x_{i}-\lambda_{i}\right)\left[\left(x-x_{i}-\lambda_{i}\right)^{2}-3 y^{2}\right]}{\left[\left(x-x_{i}-\lambda_{i}\right)^{2}+y^{2}\right]^{3}} \eta_{i}$,

$\sigma_{x y}=-2 B_{x} \sum_{i=2}^{n} \frac{\left(x-x_{i}-\lambda_{i}\right)^{4}+y^{4}-6\left(x-x_{i}-\lambda_{i}\right)^{2} y^{2}}{\left[\left(x-x_{i}-\lambda_{i}\right)^{2}+y^{2}\right]^{3}} \eta_{i}$.

Die Koordinatentransformation ist durch

$y=\cos \alpha y^{\prime}-\sin \alpha x^{\prime}=\frac{1}{3} y^{\prime}-\frac{2 \sqrt{2}}{3} x^{\prime}$,

$\eta_{1}+x=\sin \alpha y^{\prime}+\cos \alpha x^{\prime}=\frac{2 \sqrt{2}}{3} y^{\prime}+\frac{1}{3} x^{\prime}$

gegeben und die Transformation der Spannungskomponenten, von denen uns nur $\sigma_{y z}^{\prime}$ und $\sigma_{x y}^{\prime}$ interessieren, durch ${ }^{21}$

$\sigma_{y z}^{\prime}=\sin \alpha \sigma_{z x}+\cos \alpha \sigma_{y z}$,

$\sigma_{x y}^{\prime}=\sin \alpha \cos \alpha\left(\sigma_{x x}-\sigma_{y y}\right)-\left(\sin ^{2} \alpha-\cos ^{2} \alpha\right) \sigma_{x y}$

oder $\quad \sigma_{y z}^{\prime}=\frac{2 \sqrt{2}}{3} \sigma_{z x}+\frac{1}{3} \sigma_{y z}$

$$
\sigma_{x y}^{\prime}=\frac{2 \sqrt{2}}{9}\left(\sigma_{x x}-\sigma_{y y}\right)-\frac{7}{9} \sigma_{x y} .
$$

21 G. Joos, Lehrbuch der Theoretischen Physik, 8. Auflage, Akademische Verlagsgesellschaft Geest \& Portig K.G., Leipzig 1954, S. 29. 


\section{Die Aktivierungsenergie für die Quergleitung}

Die Aktivierungsenergie $U$ (siehe Einleitung) setzt sich zusammen aus der Änderung der Selbstenergie $\Delta E^{\mathrm{AA}}$ der Halbversetzung A (Abb. 2), der Änderung der Wechselwirkungsenergie $\Delta E^{\mathrm{AB}}$ der Halbversetzungen $\mathrm{A}$ und $\mathrm{B}$, der freiwerdenden Stapelfehlerenergie $\Delta E_{\mathrm{St}}$ und der von der äußeren Spannung geleisteten Arbeit $W$,

$$
U=\Delta E^{\mathrm{AA}}+\Delta E^{\mathrm{AB}}-\Delta E_{\mathrm{St}}-W .
$$

Kröner, SeEger und Wolf ${ }^{8}$ haben die Selbstenergie $E^{\mathrm{AA}}$ und die Wechselwirkungsenergie $E^{\mathrm{AB}}$ der Versetzungen $A$ und $B$ für die aufgespaltene und für die partiell rekombinierte Konfiguration nach KRÖNER ${ }^{9}$ berechnet. Wir übernehmen, indem wir die Differenzen bilden, $\Delta E^{\mathrm{AA}}$ und $\Delta E^{\mathrm{AB}}$ von dort.

$\Delta E_{\mathrm{St}}$ ist gleich dem Produkt aus spezifischer Stapelfehlerenergie $\gamma$ und der Flächenverminderung $\Delta F$ des Stapelfehlerbandes durch die Rekombination,

$$
\Delta E_{\mathrm{St}}=\gamma \cdot \Delta F \text {. }
$$

Die äußere Spannung leistet bei der Rekombination Arbeit. Diese ist gegeben durch

$$
W=\int_{\Delta F} K_{\mathrm{F}} \mathrm{d} x \mathrm{~d} z
$$

Die Kraft $K_{\mathrm{F}}$ ist unabhängig von $z$. Die numerische Rechnung zeigt, daß sie sich auch im Variationsbereich von $x$ nur wenig ändert, so daß man

$$
W=K_{\mathrm{F}} \Delta F
$$

setzen kann, wobei

$$
\begin{aligned}
\Delta F=4 \eta^{\prime}(l+ & \left.\xi \ln \frac{L}{\xi}\right) \\
& =4 \eta\left(1-\frac{\delta}{\eta}\right)\left(l+\xi \ln \frac{L}{\xi}\right), \quad \eta^{\prime}=\eta-\delta
\end{aligned}
$$

ist, wenn $\mathrm{A}_{2}$ in Abb. 2 durch eine Hyperbel mit der Gleichung

$$
x=2 \eta^{\prime}-\frac{2 \eta^{\prime} \xi}{z-l+\xi}
$$

beschrieben wird. Aus den Gln. (1.5) und (1.14) folgt

$$
K_{\mathrm{F}}+\gamma=\frac{b B}{8}\left(1-\frac{\mu}{3}\right) \frac{1}{\eta}
$$

und damit wird

$$
\Delta E_{\mathrm{St}}+W=\frac{b B}{2}\left(1-\frac{\mu}{3}\right)\left(1-\frac{\delta}{\eta}\right)\left(l+\xi \ln \frac{L}{\xi}\right) .
$$

Das Gesamtergebnis wird zusammengeschrieben, indem die einzelnen Größen aus ${ }^{8}$ übernommen und entsprechende Ausdrücke geeignet zusammengefaßt werden. Nach Kröner kann man die Selbstenergie als folgende Summe schreiben

$$
\begin{gathered}
\Delta E^{\mathrm{AA}}=b_{x}^{\mathbf{A}^{2}} \Delta M_{x x}^{\mathrm{AA}}+b_{z}^{\mathbf{A}^{2}} \Delta M_{z z}^{\mathrm{AA}}, \quad b_{x}^{\mathrm{A}}=-\frac{b}{2 \sqrt{3}}, \\
b_{z}^{\mathbf{A}}=\frac{b}{2} .
\end{gathered}
$$

Die Selbstinduktivitäten $M_{i i}^{\mathrm{AA}}$ werden als doppelte Linienintegrale berechnet, wobei zweimal über die Versetzungslinie A integriert wird.

$$
\begin{gathered}
\Delta M_{i i}^{\mathrm{AA}}=\left(2 M_{i i}^{\mathrm{A}_{2} \mathrm{~A}_{2}}+M_{i i}^{\mathrm{A}_{3} \mathrm{~A}_{3}}-M_{i i}^{\mathrm{A} \overline{\mathrm{A}}}\right)+\left(M_{i i}^{\mathrm{A}_{1} \mathrm{~A}_{2}}+2 M_{i i}^{\mathrm{A}_{1} \mathrm{~A}_{3}}\right), \quad i=x, z \\
2 M_{i i}^{\mathrm{A}_{2} \mathrm{~A}_{2}}+M_{i i}^{\mathrm{A}_{3} \mathrm{~A}_{3}}-M_{i i}^{\overline{\mathrm{A}} \overline{\mathrm{A}}}=\frac{G}{8 \pi}\left(\sum_{k=0,2}^{\infty}\left(D_{i i}^{k}-E_{i i}^{k+2}\right) I_{x^{\prime} x}^{k}+E_{i i}^{0} I\right) \\
I_{x^{\prime} x}^{k}=2 \eta^{\prime}\left\{\alpha^{k+1}\left(\frac{1}{2 k+3} \ln \frac{\left(2 \eta^{\prime}\right)^{2}+\xi^{2}}{\varepsilon^{2}}-\frac{1}{2 k+3}\left(\frac{2}{2 k+3}+\sum_{p=1}^{2 k+2} \frac{1}{p}+2 \sum_{r=1}^{k+1} \frac{1}{r}\right)+\sum_{r=1}^{k+1} \frac{1}{r(2 k+3-r)}\right)\right. \\
\left.\Phi=2 \operatorname{arctg} \frac{4}{2 k+3} \sum_{j=2,4}^{k} \frac{(-1)(k-j) / 2}{2 j-1} \alpha^{j-1}+\frac{(-1) k / 2}{2 k+3} \frac{1}{\sqrt{2 \alpha}}\left(\ln \frac{1-\sqrt{2 \alpha}+\alpha}{1+\sqrt{2 \alpha}+\alpha}+\Phi\right)\right\} \\
\Phi=2\left(\pi-\operatorname{arctg} \frac{\sqrt{2 \alpha}}{\alpha-1}\right), \quad \alpha<1, \quad \alpha>1, \quad \alpha=\frac{2 \eta^{\prime}}{\xi} ; \\
\Phi=\pi, \quad \alpha=1, \quad 3.12) \\
I=-\left(2 L \ln 2+2 l\left(\ln \frac{L}{2 l}+1\right)\right)-\xi \ln \left(\alpha^{2}+1\right)+\frac{2 \eta^{\prime}}{\sqrt{2 \alpha}}\left(\ln \frac{1-\sqrt{2 \alpha}+\alpha}{1+\sqrt{2 \alpha}+\alpha}+\Phi\right)
\end{gathered}
$$




$$
\begin{gathered}
M_{i i}^{\mathrm{A}_{1} \mathrm{~A}_{2}}+2 M_{i i}^{\mathrm{A}_{1} \mathrm{~A}_{3}}=\frac{G}{8 \pi} \sum_{k=0,2}^{\infty}\left\{\left(-\left(\frac{2 \eta^{\prime}}{\lambda \xi}\right)^{2} D_{i i}^{k}+E\right.\right. \\
J_{\mathrm{g}^{\prime}{ }_{1} \mathrm{~g}_{1}}^{0}=2(l+\lambda \xi) \ln \frac{2(l+\lambda \xi)}{2 l+\lambda \xi}+2 l \ln \frac{2 l}{2 l+\lambda \xi}, \\
J_{\mathrm{g}^{\prime}{ }_{1} \mathrm{~g}_{1}}^{2}=\left(\frac{2 \eta^{\prime}}{\lambda \xi}\right)^{2}\left(J_{\mathrm{g}^{\prime} \mathrm{g}_{1}}^{0}-\frac{(\lambda \xi)^{2}}{2 l+\lambda \xi}\right), \quad(3.17) \\
2 J_{\mathrm{g}^{\prime}{ }^{\prime} \mathrm{g}_{1}}^{0}+J_{\mathrm{g}^{\prime} \mathrm{g}_{2}}^{0}+2 J_{\mathrm{A}_{3} \mathrm{~A}_{1}}^{0}=\left(2 L \ln 2+2 l\left(\ln \frac{L}{2 l}+1\right)\right) \\
+2\left(l \ln \frac{l+\lambda \xi}{l}-(2 l+\lambda \xi) \ln \frac{2(l+\lambda \xi)}{2 l+\lambda \xi}\right), \quad(3.18) \\
2 J_{\mathrm{g}_{2} \mathrm{~g}_{1}}^{2}+J_{\mathrm{g}^{\prime} \mathrm{g}_{2}}^{2}+2 J_{\mathrm{A}_{3} \mathrm{~A}_{1}}^{2} \\
=\left(\frac{2 \eta^{\prime}}{\lambda \xi}\right)^{2}\left(\frac{(\lambda \xi)^{2}}{2 l+\lambda \xi}+2\left(\lambda \xi-J_{\mathrm{g}_{1} \mathrm{~g}_{1}}^{0}\right)\right), \quad \lambda \approx 2, \\
\varepsilon=0,3 b\left(\mu \sin ^{2} \beta+\cos ^{2} \beta\right) . \quad(3.20)
\end{gathered}
$$

$\beta$ ist der Winkel zwischen Versetzungslinie und Burgers-Vektor.

$$
\begin{aligned}
D_{x x}^{k} & =a_{k}+2 a_{k-2}, \quad E_{x x}^{k}=2 \mu a_{k}+(2 \mu+1) a_{k-2}, \\
D_{z z}^{k} & =(2 \mu+1) a_{k}+2 \mu a_{k-2}, \quad E_{z z}^{k}=2 a_{k}+a_{k-2}, \\
a_{0} & =\frac{23}{2}, \quad a_{2}=-\frac{16}{2}, \quad a_{4}=\frac{10}{81} .
\end{aligned}
$$

Die Wechselwirkungsenergie erhält man ähnlich wie die Selbstenergie als Summe

$$
\begin{array}{r}
\Delta E^{\mathrm{AB}}=b_{x}^{\mathrm{A}} b_{x}^{\mathrm{B}} \Delta M_{x x}^{\mathrm{AB}}+b_{z}^{\mathrm{A}} b_{z}^{\mathrm{B}} \Delta M_{z z}^{\mathrm{AB}}, \quad \\
b_{x}^{\mathrm{B}}=\frac{b}{2 \sqrt{3}}, b_{z}^{\mathrm{B}}=\frac{b}{2},
\end{array}
$$

wobei die Gegeninduktivitäten $M_{i i}^{\mathrm{AB}}$ wieder als doppelte Linienintegrale berechnet werden. Integriert wird einmal über die Versetzungslinie A und einmal über B.

$$
\begin{gathered}
\Delta M_{i i}^{\mathrm{AB}}=2 M_{i i}^{\mathrm{A}_{1} \mathrm{~B}}+M_{i i}^{\mathrm{A}_{3} \mathrm{~B}}-M_{i i}^{\mathrm{AB}}, \\
\Delta M_{z z}^{\mathrm{AB}}=\frac{G}{\pi}\left(l \ln \frac{\eta}{\delta}+\xi\left(\left(1-\frac{\delta}{\eta}\right)\left(\ln \frac{L}{\xi}+1\right)\right.\right. \\
\left.\left.+\frac{\delta}{\eta} \ln \frac{\delta}{\eta}\right)\right), \\
\Delta M_{x x}^{\mathrm{AB}}=\mu \Delta M_{z z}^{\mathrm{AB}} .
\end{gathered}
$$

Das ergibt, wenn wir $\Delta E^{\mathrm{AB}}$ und $\Delta E_{\mathrm{St}}+W$ zusammenfassen,

$$
\begin{aligned}
& \Delta E^{\mathrm{AB}}- \Delta E_{\mathrm{St}}-W=\frac{G b^{2}}{4 \pi}\left(1-\frac{\mu}{3}\right) \\
& \cdot\left(l\left[\ln \frac{\eta}{\delta}-1+\frac{\delta}{\eta}\right]+\xi\left[1-\frac{\delta}{\eta}\left(1+\ln \frac{\eta}{\delta}\right)\right]\right) .
\end{aligned}
$$

als Funktion von $l$ sucht. $E_{\mathrm{I}}=U$ ist bekannt, $E_{\mathrm{II}}$ wird im folgenden berechnet.

Gl. (3.25) enthält die Länge $L$ der Versetzung nicht mehr. In der Selbstenergie $\Delta E^{\mathrm{AA}}$ tritt sie jedoch auf. Es ist leicht einzusehen, daß sich die Ausdrücke mit $L$ bei der Summation gegenseitig wegheben. Damit wird, wie zu erwarten war, die Aktivierungsenergie für die Quergleitung unabhängig von $L$.

Die obigen Formeln wurden im Rahmen der linearen Elastizitätstheorie gewonnen. Das hatte zur Folge, daß, um zu vermeiden, daß bei der Rekombination der beiden Halbversetzungen die Energie unendlich groß wird, ein kleinster Abstand $2 \delta$ eingeführt werden mußte (Abb. 2), der noch unbestimmt ist. Man gewinnt diese Größe, indem man fordert, daß die Energie der Dissoziation einer geradlinigen vollständigen Versetzung in zwei parallele geradlinige Halbversetzungen im linearen Modell gleich der Dissoziationsenergie $E_{\text {dis }}$ ist, die von SEEgER und Sснӧск ${ }^{18}$ im Peierlsschen Modell berechnet wurde,

$$
E_{\mathrm{dis}}=\frac{G b^{2}}{8 \pi}\left(1-\frac{\mu}{3}\right) \ln \frac{\eta_{0}}{\delta}-2\left(\eta_{0}-\delta\right) \gamma .
$$

Für die Versetzungslinien $A_{1}$ und $A_{2}$ der gekrümmten Versetzung A wurden Hyperbelstücke gewählt mit dem freien Parameter $\xi$, der die Kurvenform bestimmt. Das gestattet, die Kurvengleichung dem wirklichen Versetzungsverlauf anzupassen, indem durch Variation von $\xi$ das Minimum der Konfigurationsenergie von Abb. 2 bestimmt wird.

\section{Die Bestimmung der kritischen Länge $2 \boldsymbol{l}_{0}$}

Wir betrachten die Energie der gesamten Anordnung von Abb. $1^{6,7}$. Bei der Quergleitung muß in der Ebene I Energie aufgewendet werden $\left(E_{\mathrm{I}}\right)$, in der Ebene II wird Energie frei $\left(E_{\mathrm{II}}\right)$. Ohne äußere Spannung ist die aufzuwendende Energie immer größer als die freiwerdende. Das heißt, ohne äußere Spannung ist diese Versetzungskonfiguration immer instabil. Die Anordnung ist dann stabil, wenn bei äußerer Spannung mit wachsendem $l$ die Zunahme von $E_{\mathrm{II}}$ größer ist als die Zunahme von $E_{\mathrm{I}}$. Die kritische Länge $2 l_{0}$ erhält man also, wenn man den Maximalwert der Energiedifferenz

$$
\Delta E=E_{\mathrm{I}}-E_{\mathrm{II}}
$$

$$
\text { wird im folgenden berechnet. }
$$


In Abb. 6 a ist die Quergleitebene in die Bildebene gelegt. Die beiden Halbversetzungsstücke C und D haben Parabelform. Wir begnügen uns hier mit einer gröberen Näherung und vernachlässigen die Änderung der Wechselwirkungsenergie von C und D mit den Versetzungsteilen in der primären Gleitebene. $E_{\mathrm{II}}$ setzt sich aus vier Anteilen zusammen, aus der Änderung der Selbstenergie $\left(\Delta E^{\mathrm{CC}}+\Delta E^{\mathrm{DD}}\right)$, der Änderung der Wechselwirkungsenergie $\left(\Delta E^{\mathrm{CD}}\right)$, der

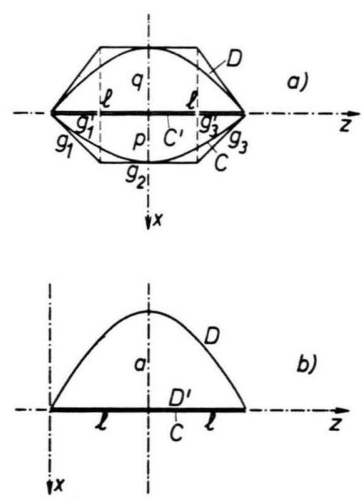

Abb. 6. Aufspaltung der Versetzung in der Quergleitebene.

Arbeit, die das Spannungsfeld leistet $\left(W_{\text {II }}\right)$, und der aufzuwendenden Stapelfehlerenergie $\left(E_{\mathrm{StII}}\right)$ bei der Aufspaltung in der Quergleitebene,

$$
E_{\mathrm{II}}=\Delta E^{\mathrm{CD}}+W_{\mathrm{II}}-\left(\Delta E^{\mathrm{CC}}+\Delta E^{\mathrm{DD}}\right)-E_{\mathrm{StII}} \cdot
$$

Die Selbst- und Wechselwirkungsenergien werden nach der KRöNerschen Theorie ${ }^{9}$ berechnet. Es wird hierbei auf die später ${ }^{8}$ ausführlich behandelte Berechnung der Energie gekrümmter Versetzungen vorgegriffen.

Zur Berechnung der Selbstenergie $E^{\mathrm{CC}}$ wird die Parabel C mit der Gleichung

$$
x=p-\frac{p}{l^{2}} z^{2}
$$

durch ihre Tangenten

$\mathrm{g}_{1}: \quad x=2 p\left(1+\frac{z}{l}\right), \quad-l \leqq z \leqq-\frac{l}{2}$,

$$
\begin{aligned}
& \mathrm{g}_{2}: \quad x=p, \quad-\frac{l}{2} \leqq z \leqq \frac{l}{2}, \\
& \mathrm{~g}_{3}: \quad x=2 p\left(1-\frac{z}{l}\right), \quad \frac{l}{2} \leqq z \leqq l
\end{aligned}
$$

ersetzt. In dem Ausdruck für die Energie, der, da der Burgers-Vektor von $\mathrm{C}$ in der Versetzungsebene liegt $\left(b_{y}=0\right)$, folgende einfache Form annimmt

$\Delta E^{\mathrm{cc}}=b_{x}^{\mathrm{c}^{2}} \Delta M_{x x}^{\mathrm{cc}}+b_{z}^{\mathrm{c}^{2}} \Delta M_{z z}^{\mathrm{cc}}+b_{x}^{\mathrm{c}} b_{z}^{\mathrm{c}}\left(\Delta M_{x z}^{\mathrm{cc}}+\Delta M_{z x}^{\mathrm{cc}}\right)$,

wird näherungsweise

$$
\begin{array}{r}
\Delta M_{i q}^{\mathrm{cc}}=M_{i q}^{\mathrm{cc}}-M_{i q}^{\mathrm{c}^{\prime} \mathrm{c}^{\prime}}=\Delta M_{i q}^{\mathrm{g}_{1} \mathrm{~g}_{1}}+\Delta M_{i q}^{\mathrm{g}_{3} \mathrm{~g}_{3}} \\
=M_{i q}^{\mathrm{g}_{1} \mathrm{~g}_{1}}-M_{i q}^{\mathrm{g}^{\prime}{ }_{1}{ }^{\prime}{ }^{\prime}{ }_{1}}+M_{i q}^{\mathrm{g}_{3} \mathrm{~g}_{3}}-M_{i q}^{\mathrm{g}^{\prime}{ }_{3} \mathrm{~g}^{\prime}{ }_{3}}
\end{array}
$$

gesetzt. Ein an anderer Stelle durchgeführter numerischer Vergleich zwischen genauer und in dieser Weise vereinfachter Rechnung zeigt, daß die Abweichung sehr gering ist ${ }^{22}$, was uns zu diesem Schritt berechtigt.

Es ist sinnvoll, die einzelnen Größen nicht getrennt zu berechnen, sondern sie $\mathrm{zu} M_{i q}^{\mathrm{g}_{1} \mathrm{~g}_{1}}+M_{i q}^{\mathrm{g}_{\mathrm{g}} \mathrm{g}_{3}}$ und $M_{i q}^{g_{i q}^{\prime} g^{\prime}{ }_{1}}+M_{i q}^{g^{\prime}{ }_{2} g^{\prime}{ }_{3}}$ zusammenzufassen. Bei einer Spiegelung an der $x$-Achse geht $g_{1}$ in $g_{3}$ und $g_{1}{ }^{\prime}$ in $\mathrm{g}_{3}{ }^{\prime}$ über. Da die Energie $\Delta E^{\mathrm{CC}}$ invariant gegen diese Transformation ist, die $z$-Komponente des BurgersVektors jedoch ihr Vorzeichen wechselt, müssen auch die $M_{x z}$ und $M_{z x}$ ihr Vorzeichen umkehren, nicht dagegen die $M_{x x}$ und $M_{z z}$. Das führt auf folgende Beziehungen

$$
\begin{aligned}
& M_{x x}^{g_{1} g_{1}}=M_{x x}^{g_{3} g_{3}}, \quad M_{x x}^{g_{1} g_{1}}+M_{x x}^{g_{3} g_{3}}=2 M_{x x}^{g_{1} g_{1}} \\
& M_{z z}^{g_{1} g_{1}}=M_{z z}^{g_{3} g_{3}}, \quad M_{z z}^{g_{1} g_{1}}+M_{z z}^{g_{3} g_{3}}=2 M_{z z}^{g_{1} g_{1}}, \\
& M_{x z}^{g_{1} g_{1}}=-M_{x z}^{g_{3} g_{3}}, M_{x z}^{g_{1} g_{1}}+M_{x z}^{g_{3} g_{3}}=M_{z x}^{g_{1} g_{1}}+M_{z x}^{g_{3} g_{3}}=0 . \\
& M_{z x}^{g_{1} g_{1}}=-M_{z x}^{g_{3} g_{3}},
\end{aligned}
$$

Dasselbe gilt für $\mathrm{g}_{1}{ }^{\prime}$ und $\mathrm{g}_{3}{ }^{\prime}$.

Wir entnehmen der Arbeit von Kröner, Seeger und WolF 8

$$
\begin{aligned}
& M_{x x}^{\mathrm{g}_{x x} \mathbf{g}_{1}}=\frac{G}{16 \pi} \int_{\mathbf{g}_{1}} \int_{\mathrm{g}^{\prime}{ }_{1 \varepsilon}}\left(2 \frac{\left(x-x^{\prime}\right)^{2}}{r^{3}} \mathrm{~d} x^{\prime} \mathrm{d} x+(2 \mu+1) \frac{\left(x-x^{\prime}\right)^{2}}{r^{3}} \mathrm{~d} z^{\prime} \mathrm{d} z+\frac{\left(z-z^{\prime}\right)^{2}}{r^{3}} d x^{\prime} \mathrm{d} x+2 \mu \frac{\left(z-z^{\prime}\right)^{2}}{r^{3}} \mathrm{~d} z^{\prime} \mathrm{d} z\right), \\
& M_{z z}^{\mathbf{g}_{1} g_{1}}=\frac{G}{16 \pi} \iint_{\mathbf{g}_{1} \mathbf{g}^{\prime}{ }^{\prime}}\left(2 \mu \frac{\left(x-x^{\prime}\right)^{2}}{r^{3}} \mathrm{~d} x^{\prime} \mathrm{d} x+\frac{\left(x-x^{\prime}\right)^{2}}{r^{3}} \mathrm{~d} z^{\prime} \mathrm{d} z+(2 \mu+1) \frac{\left(z-z^{\prime}\right)^{2}}{r^{3}} \mathrm{~d} x^{\prime} \mathrm{d} x+2 \frac{\left(z-z^{\prime}\right)^{2}}{r^{3}} \mathrm{~d} z^{\prime} \mathrm{d} z\right) .
\end{aligned}
$$

$x, x^{\prime}, z, z^{\prime}$ sind die Koordinaten der Geraden $g_{1}$, $r$ ist der Abstand zweier Punkte auf $\mathrm{g}_{1} \cdot \mathrm{g}_{1 \varepsilon}^{\prime}$ bedeutet, daß, um zu vermeiden, daß das Integral divergiert, bei der ersten Integration über $\mathrm{g}_{1}$ das Stück $r \leqq \varepsilon$ von der Integration auszunehmen ist. Mit der Gleichung von $g_{1}(4.4)$ wird

22 unveröffentlicht. 


$$
\begin{aligned}
& M_{x x}^{\mathrm{g}_{1} \mathrm{~g}_{1}}=\frac{G}{8 \pi}\left(\left(\frac{2 p}{l}\right)^{4}+(\mu+1)\left(\frac{2 p}{l}\right)^{2}+\mu\right) \frac{1}{V(2 p / l)^{2}+1^{3}} \int_{\mathrm{g}_{1}} \int_{\mathrm{g}^{\prime}{ }_{1 \varepsilon}} \frac{\mathrm{d} z^{\prime} \mathrm{d} z}{\left|z-z^{\prime}\right|}, \\
& M_{z z}^{\mathrm{g}_{1} \mathrm{~g}_{1}}=\frac{G}{8 \tau}\left(\mu\left(\frac{2 p}{l}\right)^{4}+(\mu+1)\left(\frac{2 p}{l}\right)^{2}+1\right) \frac{1}{\sqrt{(2 p / l)^{2}+1}} \int_{\mathrm{g}_{1}} \int_{\mathrm{g}^{\prime}{ }_{1 \varepsilon}} \frac{\mathrm{d} z^{\prime} \mathrm{d} z}{\left|z-z^{\prime}\right|} .
\end{aligned}
$$

Das Integral ergibt

$$
\iint_{\mathrm{g}_{1}} \frac{\mathrm{d} z^{\prime} \mathrm{d} z}{\left|z-z^{\prime}\right|}=l\left(\ln \frac{l}{2 \varepsilon_{z}}-1\right)=l\left(\ln \frac{l \sqrt{(2 p / l)^{2}+1}}{2 \varepsilon}-1\right) \text {. }
$$

Damit wird

$$
\begin{aligned}
& \Delta M_{x x}^{\mathrm{g}_{1} \mathrm{~g}_{1}}=\frac{G}{8 \pi} l\left[\frac{1}{\sqrt{(2 p / l)^{2}+1^{3}}}\left(\left(\frac{2 p}{l}\right)^{4}+(\mu+1)\left(\frac{2 p}{l}\right)^{2}+\mu\right)\left(\ln \frac{l \sqrt{(2 p / l)^{2}+1}}{2 \varepsilon}-1\right)-\mu\left(\ln \frac{l}{2 \varepsilon}-1\right)\right], \\
& \Delta M_{z z}^{\mathrm{g}_{1} \mathrm{~g}_{1}}=\frac{G}{8 \pi} l\left[\frac{1}{\sqrt{ }(2 p / l)^{2}+1^{3}}\left(\mu\left(\frac{2 p}{l}\right)^{4}+(\mu+1)\left(\frac{2 p}{l}\right)^{2}+1\right)\left(\ln \frac{l \sqrt{(2 p / l)^{2}+1}}{2 \varepsilon}-1\right)-\left(\ln \frac{l}{2 \varepsilon}-1\right)\right],
\end{aligned}
$$

und mit $b_{x}{ }^{\mathrm{C}}=-b / 2 \sqrt{3}, b_{z}{ }^{\mathrm{C}}=b / 2$ ist

$$
\Delta E^{\mathrm{CC}}=\frac{b^{2}}{2}\left(\Delta M_{z z}^{\mathrm{g}_{1} \mathrm{~g}_{1}}+\frac{1}{3} \Delta M_{x x}^{\mathrm{g}_{1} \mathrm{~g}_{1}}\right) .
$$

Wenn man in den Gln. (4.13) $p$ durch $q$ ersetzt, crhält man die Änderung $\Delta E^{\mathrm{DD}}$ der Selbstenergie der Versetzung D.

Die Änderung $\Delta E^{\mathrm{CD}}$ der Wechselwirkungsenergie von $\mathrm{C}$ und $\mathrm{D}$ wird näherungsweise berechnet, indem $\mathrm{C}$ als Gerade und D als Parabel angenommen wird (Abb. 6 b). Es ist

$$
\begin{aligned}
E^{\mathrm{CD}}=b_{x}^{\mathrm{C}} b_{x}^{\mathrm{D}} M_{x x}^{\mathrm{CD}}+b_{z}^{\mathrm{C}} & b_{z}^{\mathrm{D}} M_{z z}^{\mathrm{CD}} \\
& +b_{x}^{\mathrm{C}} b_{z}^{\mathrm{D}} M_{x z}^{\mathrm{CD}}+b_{z}^{\mathrm{C}} b_{x}^{\mathrm{D}} M_{z x}^{\mathrm{CD}} .
\end{aligned}
$$

Bei einer Spiegelung an der Parabelachse gehen C und $\mathrm{D}$ in sich über, d. h. die $M_{i q}$ bleiben dieselben. Da hierbei die Wechselwirkungsenergie nicht geändert wird, die $z$-Komponenten der Burgers-Vektoren aber ihre Vorzeichen wechseln, muß

$$
M_{x z}^{\mathrm{CD}}=M_{z x}^{\mathrm{CD}}=0
$$

sein.

In den Formeln für die Gegeninduktivitäten zweier Versetzungen kann man eine Integration elementar ausführen, wenn beide Versetzungen in einer Ebene liegen und eine davon geradlinig ist. Läßt man die gerade Versetzung, wie in unserem Beispiel C, mit der $z$-Achse zusammenfallen, so findet man folgende Ausdrücke ${ }^{8,9}$

$$
\begin{gathered}
M_{x x}^{\mathrm{CD}}=-\frac{G}{2 \pi} \mu \int_{\mathrm{D}}\left(\ln \frac{|x|}{2 l}+\frac{1}{2}\right) \mathrm{d} z, \\
M_{z z}^{\mathrm{CD}}=-\frac{G}{2 \pi} \int_{\mathrm{D}}\left(\ln \frac{|x|}{2 l}+1\right) \mathrm{d} z .
\end{gathered}
$$

Ge'ht man mit der Gleichung der Parabel

$$
x=\frac{a z}{l^{2}}(z-2 l), \quad a=p+q,
$$

in den Integranden ein, so ergibt sich

$$
\begin{aligned}
& M_{x x}^{\mathrm{CD}}=\frac{G}{\pi} \mu l\left(\ln \frac{l}{2 a}+\frac{3}{2}\right), \\
& M_{z z}^{\mathrm{CD}}=\frac{G}{\pi} l\left(\ln \frac{l}{2 a}+1\right) .
\end{aligned}
$$

Diese Ausdrücke werden von den Gegeninduktivitäten $^{8}$

$$
\begin{aligned}
& M_{x x}^{\mathrm{CD}^{\prime}}=\frac{G}{\pi} \mu l\left(\ln \frac{l}{\delta}-\frac{1}{2}\right), \\
& M_{z z}^{\mathrm{CD}^{\prime}}=\frac{G}{\pi} l\left(\ln \frac{l}{\delta}-1\right)
\end{aligned}
$$

subtrahiert. Damit wird

$$
\begin{aligned}
& \Delta M_{x x}^{\mathrm{CD}}=\frac{G}{\pi} \mu l\left(\ln \frac{2 a}{\delta}-2\right), \\
& \Delta M_{z z}^{\mathrm{CD}}=\frac{G}{\pi} l\left(\ln \frac{2 a}{\delta}-2\right),
\end{aligned}
$$

und mit $b_{x}{ }^{\mathrm{D}}=b / 2 \sqrt{3}, b_{z}{ }^{\mathrm{D}}=b / 2$ ist

$$
\Delta E^{\mathrm{CD}}=\frac{G b^{2}}{4 \pi}\left(1-\frac{\mu}{3}\right) l\left(\ln \frac{2 a}{\delta}-2\right)
$$

Die Schubspannung in der Quergleitebene ( $\$ 2$ ) übt auf die Versetzungsteile C und D Kräfte aus und leistet damit bei der Aufspaltung in der Quergleitebene Arbeit. Da sie sich in dem uns interessierenden Bereich nur wenig ändert, können wir sie für die Energieberechnung als konstant ansehen. Sind $K_{\mathrm{C}}$ und $K_{\mathrm{D}}$ die Kräfte, die auf $\mathrm{C}$ und $\mathrm{D}$ wirken, $F_{\mathrm{C}}$ und $F_{\mathrm{D}}$ die Flächen, die von $\mathrm{C}$ und $\mathrm{D}$ bei der Aufspaltung überstrichen we: len, so ist

$$
\begin{array}{ll}
W_{\mathrm{C}}=K_{\mathrm{C}} F_{\mathrm{C}}, & F_{\mathrm{C}}=\frac{4}{3} l p, \\
W_{\mathrm{D}}=K_{\mathrm{D}} F_{\mathrm{D}}, & F_{\mathrm{D}}=\frac{4}{3} l q,
\end{array}
$$


und $\quad W_{\mathrm{II}}=W_{\mathrm{D}}-W_{\mathrm{C}}=\frac{4}{3} l\left(q K_{\mathrm{D}}-p K_{\mathrm{C}}\right)$,

wobei $K_{\mathrm{C}}$ und $K_{\mathrm{D}}$ durch

$$
\begin{aligned}
& K_{\mathrm{C}}=-\frac{b}{2} \sigma_{y z}^{\prime}+\frac{b}{2 \sqrt{3}} \sigma_{x y}^{\prime}, \\
& K_{\mathrm{D}}=-\frac{b}{2} \sigma_{y z}^{\prime}-\frac{b}{2 \sqrt{3}} \sigma_{x y}^{\prime}
\end{aligned}
$$

gegeben sind.

Mit der Stapelfehlerenergie

$$
E_{\mathrm{StII}}=\gamma\left(F_{\mathrm{C}}+F_{\mathrm{D}}\right)=\frac{4}{3} a l \gamma
$$

ist die gesamte Energie $E_{\mathrm{II}}$ gegeben. Die Parameter $p$ und $q$ bestimmt man, indem man sie bei gegebener äußerer Spannung und festem $l$ variiert und den Maximalwert von $E_{\text {II }}$ sucht. Durch Variation von $l$ wird das Maximum von $E_{\mathrm{I}}-E_{\mathrm{II}}$ und damit die kritische Länge $2 l_{0}$ bestimmt.

\section{Ergebnisse und Diskussion}

Die umfangreiche numerische Rechnung wurde für die spezifischen Stapelfehlerenergien $\gamma / G b=1,84$; 3,$69 ; 5,53 ; 7,37 \cdot 10^{-3}$ für $n=20$ und 40 durchgeführt. Abb. 7 zeigt die Abhängigkeit der kritischen Länge $2 l_{0}$ von der äußeren Spannung $\tau$. Die kritische Länge nimmt linear mit dem Logarithmus der

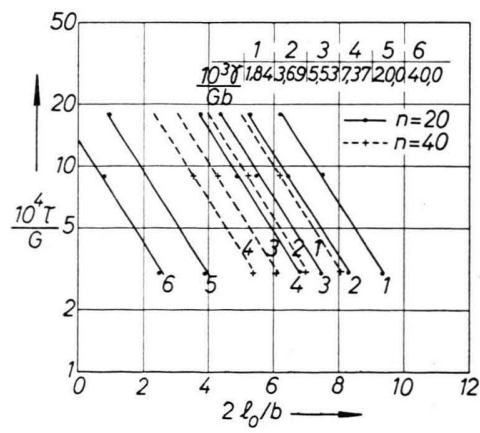

Abb. 7. Die kritische Länge als Funktion der äußeren Spannung.

äußeren Spannung ab. Die Geraden in Abb. 7 sind für verschiedene Stapelfehlerenergien und für verschiedene Werte von $n$ nahezu parallel.

Die Abhängigkeit der Aktivierungsenergie von der äußeren Spannung ist in Abb. 3 dargestellt. Für große Spannungen, d. h. kleine Aufspaltungen, gilt die lineare Krönersche Theorie nicht mehr. Vor allen Dingen liefert sie einen zu großen Wert für die Spannung, die nötig ist, um die Aufspaltung aufzuheben, d. h., bei der die Aktivierungsenergie Null wird. Die in Abb. 8 eingezeichneten Spannungswerte für $U=0$ wurden mit der von SEEGER ${ }^{23}$ aus der $\mathrm{Ab}$ hängigkeit der Versetzungsenergie von der Aufspaltung im Peierlsschen Modell ${ }^{18}$ abgeleiteten Beziehung

berechnet.

$$
\tau_{\mathrm{III}}(0)=\frac{2 G}{n}\left(0,056-\frac{\gamma}{G b}\right)
$$

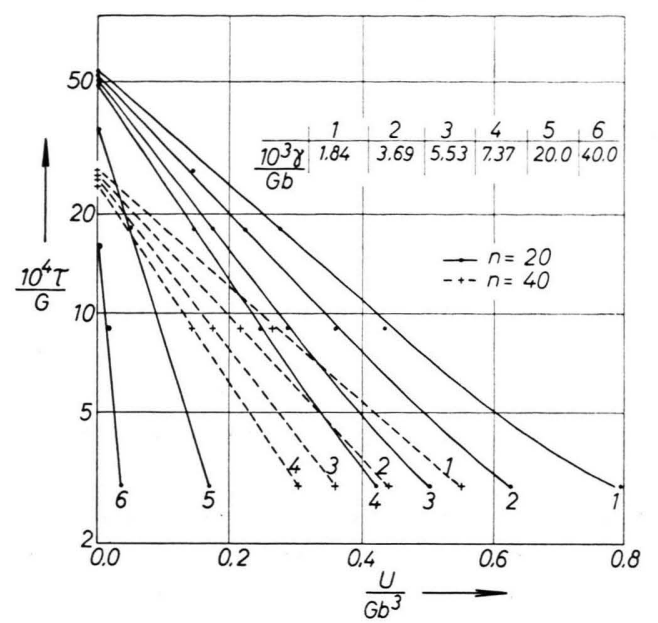

Abb. 8. Die Aktivierungsenergie für die Quergleitung als Funktion der äußeren Spannung.

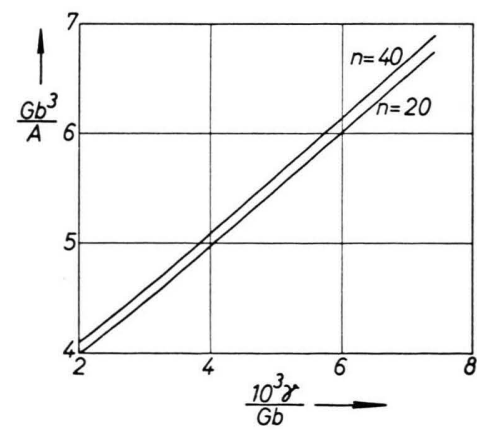

Abb. 9. Abhängigkeit des Proportionalitätsfaktors $A$ von der Stapelfehlerenergie $\gamma$ in reziproker Darstellung für kleine Werte von $\gamma$.

Die Kurven in Abb. 8 zeigen eine nahezu logarithmische Abhängigkeit der Aktivierungsenergie von der äußeren Spannung, die durch die Beziehung

$$
U=-A \ln \frac{\tau}{\tau_{\mathrm{III}}(0)}
$$

ausgedrückt werden kann. Wie Abb. 9 zeigt, ist $1 / A$ eine lineare Funktion der spezifischen Stapelfehler-

23 A. Seeger, Dislocations and Mechanical Properties of Crystals, J. Wiley \& Sons, New York 1957, S. 243. 
energie $\gamma$. Man sieht, da $\beta A$, wie zu erwarten war, nur wenig von $n$ abhängt, daß also die $U(\ln \tau)$-Geraden in Abb. 8 für festes $\gamma$ und verschiedenes $n$ nahezu parallel sind. Dieser einfache Zusammenhang gestattet, wenn zwischen den beiden $n$-Werten linear interpoliert wird, das Gesamtergebnis in folgender geschlossenen Form darzustellen

$$
\begin{gathered}
U=-\frac{0,352 G b^{3}}{\left(1+\frac{n}{900}\right)\left(1+\frac{180 \gamma}{G b}\right)} \ln \frac{\tau}{\tau_{\mathrm{III}}(0)} \\
=-\frac{0,352 G b^{3}}{\left(1+\frac{n}{900}\right)\left(1+\frac{2,87 b}{2 \eta_{0}}\right)} \ln \frac{\tau}{\tau_{\mathrm{III}}(0)} .
\end{gathered}
$$

Für große spezifische Stapelfehlerenergien, d. h. kleine Aufspaltungen, gelten die im Rahmen der linearen Elastizitätstheorie gewonnenen Formeln (5.3) nicht. Für diesen Bereich wurde auf die Rechnung von SchöcK und $\mathrm{S}_{\text {EEgER }}{ }^{7}$ und $\mathrm{S}_{\text {снӧс⿰K}}{ }^{6}$ zurückgegriffen. Das Ergebnis der auf Versetzungsaufstauungen erweiterten Rechnung ist in Abb. 8 mit aufgetragen. Die Kurven für $\gamma / G b=20$ und $40 \cdot 10^{-3}$ zeigen ebenfalls eine logarithmische Abhängigkeit der Aktivierungsenergie von der äußeren Spannung. In Abb. 10 ist für den ganzen Stapelfehlerenergiebereich $\mathrm{A}$ als Funktion von $\gamma$ dargestellt. A wird Null für $\gamma / G b=56 \cdot 10^{-3}$. Die Ergebnisse der bei-

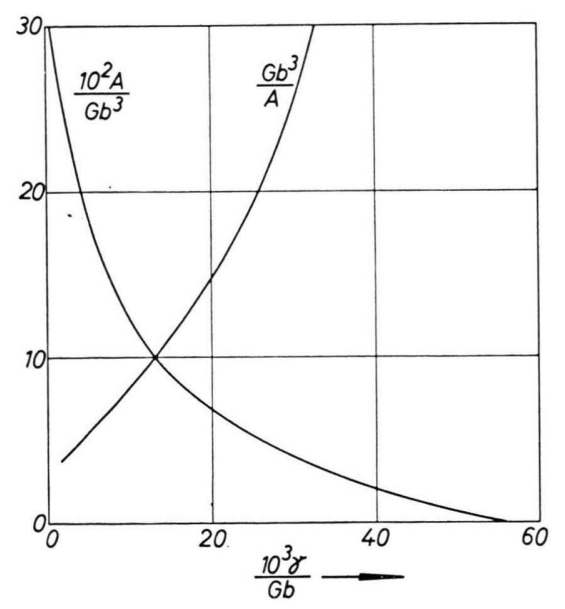

Abb. 10. Abhängigkeit des Proportionalitätsfaktors $A$ von der Stapelfehlerenergie $\gamma$ für den ganzen interessierenden Stapelfehlerenergiebereich.

24 bei A. SEEger, Hand. d. Physik VII/1, Springer-Verlag, Berlin-Göttingen-Heidelberg 1955, S. 609, mit $G_{\text {st }}$ bezeichnet.

25 Eine untere Grenze für $\gamma$ kann man aus dem Auftreten bzw. Nichtauftreten von Stapelfehlern bei der Kondensation von Leerstellen in abgeschreckten Metallen erhalten. den verschiedenen Rechenmethoden schließen sich sehr gut aneinander an, was anzeigt, daß das verwendete Modell keine schlechte Näherung darstellt. Da in dem verwendeten Maßstab die Kurven für verschiedene $n$ fast zusammenfallen, haben wir sie für $n=20$ gezeichnet. Die Kurven enthalten außerdem die Porssonsche Konstante $v$ als Parameter. Die Abhängigkeit ist gering. Der Rechnung liegen die bei Kupfer unter näherungsweiser Berücksichtigung der Anisotropie ${ }^{18}$ zutreffenden Verhältnisse zugrunde. In der Ordinate hat man als Schubmodul den die elastische Wechselwirkung von Schraubenversetzungen in der $\{111\}$-Ebene bestimmenden Modul $G_{2}$ (bei SEEGer und $S_{\text {ChöcK }}{ }^{18}$ mit $1 / K_{2}$ bezeichnet), in der Abszisse den bei der Scherung der $\{111\}$-Ebenen wirksamen Modul ${ }^{24} \mathrm{G}_{3}$ einzusetzen.

Eine logarithmische Abhängigkeit der Aktivierungsenergie von der Spannung wurde schon bei der einfacheren Rechnung von ScHöck und $\mathrm{SEEGER}^{7}$ gefunden. HaAsen ${ }^{3}$ hat gezeigt, daß dieses Ergebnis eine Erklärung der experimentell gefundenen exponentiellen Temperaturabhängigkeit der Spannung $\mathbf{4 , 5}$ $\tau_{\text {III, }}$ bei der Bereich III beginnt, gestattet. Der $\mathrm{Zu}$ sammenhang zwischen der Spannung $\tau_{\text {III }}$ und der Temperatur $T$ ist durch

$$
\ln \frac{\tau_{\mathrm{III}}(T)}{\tau_{\mathrm{III}}(0)}=\frac{k T}{A} \ln \frac{\dot{a}}{\dot{a}_{0}}
$$

gegeben, wo $k$ die Boltzmannsche Konstante und $\dot{a}$ die Abgleitgeschwindigkeit bedeutet. SEeger, Berner und Wolf ${ }^{5}$ haben auf Grund dieser Beziehung mit Hilfe der vorliegenden Rechenergebnisse aus Messungen der Abhängigkeit der Spannung $\tau_{\text {III }}$ von der Temperatur und der Abgleitgeschwindigkeit die spezifischen Stapelfehlerenergien einiger kubisch-flächenzentrierter Metalle bestimmt ${ }^{25}$. Die Bedeutung der vorliegenden Arbeit liegt darin, daß sie diese Bestimmung ermöglicht hat.

In der Einleitung wurde darauf hingewiesen, daß das der vorliegenden Rechnung zugrunde gelegte Modell nur als Näherung betrachtet werden darf, daß aber diese Näherung auf Grund der ausführlichen Diskussion von SeEger, Berner und Wolf ${ }^{5}$ als gerechtfertigt erscheint. Eine weitere Stütze erhält unser Modell durch Messungen von Berner ${ }^{26}$. Ber-

Herr Dr. J. Eshelby hat freundlicherweise darauf aufmerksam gemacht, daß in den diesbezüglichen Formeln bei Seeger, Berner und $W_{\text {olf }}{ }^{5}$ ein Fehler enthalten ist. In den dortigen Gln. (9a) und (10) sowie in Abb. 7 ist $\gamma$ durch $2 \gamma \mathrm{zu}$ ersetzen. Die dortigen Angaben über die Stapelfehlerenergien werden dadurch nicht beeinflußt.

26 R. Berner, Dissertation TH Stuttgart 1960. 
NER hat bei verschiedenen Temperaturen die Abhängigkeit der Spannung $\tau_{\text {III }}$ von der Abgleitgeschwindigkeit bestimmt und bei allen Temperautren denselben Wert für den Proportionalitätsfaktor $A$ gefunden, der aus diesen Messungen gewonnen wird. Das beweist, daß die ursprünglich ${ }^{5}$ diskutierte Alternativmöglichkeit, daß bei verschiedenen Temperaturen verschiedene Mechanismen mit verschiedenen Aktivierungsenergien die Quergleitung hervorrufen, nicht zutrifft.

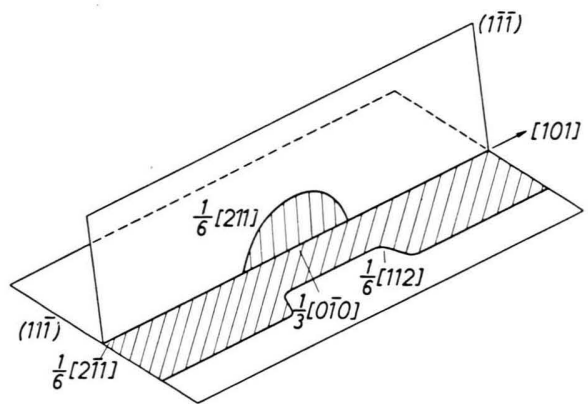

Abb. 11. Quergleitung einer aufgespaltenen Schraubenversetzung nach F LEISCHER.

Es soll noch kurz das von FleISCHER ${ }^{12}$ vorgeschlagene Modell für die Quergleitung diskutiert werden. In Abb. 11 sind die primäre Gleitebene und die Quergleitebene als (111) - und (111)-Ebenen gezeichnet. Die Schraubenversetzung mit dem Burgers-Vektor $\frac{1}{2}[101]$ ist in die Halbversetzungen $\frac{1}{6}[211]$ und $\frac{1}{6}[112]$ aufgespalten. Fleischer nimmt an, daß Quergleitung erfolgt, indem die vordere Versetzung ( $\left.\frac{1}{6}[211]\right)$ in zwei Halbversetzungen aufspaltet,

$$
\frac{1}{6}[211] \rightarrow \frac{1}{6}[211]+\frac{1}{3}[010],
$$

von denen die eine $\left(\frac{1}{6}[211]\right)$ in der Quergleitebene wandert, und die andere $\left(\frac{1}{3}[010]\right)$, da sie unbeweglich ist, in der Schnittlinie der primären Gleitebene und der Quergleitebene festgehalten wird, und da $\beta$ darauf die zweite Halbversetzung in der primären Gleitebene $\left(\frac{1}{6}[112]\right)$ von der unbeweglichen Versetzung angezogen wird und sich mit ihr vereinigt,

$$
\frac{1}{6}[112]+\frac{1}{3}[0 \overline{1} 0] \rightarrow \frac{1}{6}[1 \overline{1} 2] .
$$

Die erste Reaktion erfordert Energie und kann deshalb nur thermisch aktiviert erfolgen. Die Energie für diesen Prozeß vergleicht Fleischer mit der Energie der thermisch aktivierten Reaktion in unserem Modell,

$$
\frac{1}{6}[112]+\frac{1}{6}[2 \overline{1} 1] \rightarrow \frac{1}{2}[101],
$$

indem er die Quadrate der Burgers-Vektoren der an den Reaktionen beteiligten Versetzungen vergleicht. Er findet, daß die Energie für (5.5) nur $\frac{2}{3}$ der für (5.7) ist. Diese Abschätzung ist sehr unsicher, da die Wechselwirkungsenergien, die Arbeit, die die äußere Spannung leistet [diese ist für (5.7) ungefähr dreimal so groß wie für (5.5) ] und die Stapelfehlerenergie nicht berücksichtigt werden. Noch fragwürdiger erscheint uns, daß sich die Konfiguration nach der Reaktion (5.5) nicht in einem Sattelpunkt befindet. Ein echter Sattelpunkt ist erst nach der zusätzlichen Reaktion (5.6) erreicht. Wird nun gefordert, daß beide Reaktionen gleichzeitig erfolgen, dann ist genau die Reaktion (5.7) gegeben. Es scheint somit, daß der Fleischersche Reaktionsweg gegenüber dem hier behandelten nichts wesentlich Neues bringt.

Herrn Professor Dr. U. Dehlinger danke ich sehr für sein förderndes Interesse an der vorliegenden Arbeit. Mein besonderer Dank gilt Herrn Prof. Dr. A. SeEger für die Anregung zu diesen Untersuchungen und für die Anleitung und fördernde Unterstützung bei der Durchführung dieser Arbeit. Herzlichen Dank sage ich auch Herrn Dr. E. KRönER für wertvolle Ratschläge. Der D e u t $\mathrm{sch}$ en Forschungsgemeinschaft danke ich für finanzielle Unterstützung. 